\title{
Nuclear matter symmetry energy and the neutron skin thickness of heavy nuclei
}

\author{
Lie-Wen Chen, ${ }^{1,2}$ Che Ming Ko, ${ }^{3}$ and Bao-An Li ${ }^{4}$ \\ ${ }^{1}$ Institute of Theoretical Physics, Shanghai Jiao Tong University, Shanghai 200240, China \\ ${ }^{2}$ Center of Theoretical Nuclear Physics, National Laboratory of Heavy Ion Accelerator, Lanzhou 730000, China \\ ${ }^{3}$ Cyclotron Institute and Physics Department, Texas A\&M University, College Station, Texas 77843-3366 \\ ${ }^{4}$ Department of Chemistry and Physics, P.O. Box 419, Arkansas State University, State University, Arkansas 72467-0419
}

(Received 5 September 2005; published 15 December 2005)

\begin{abstract}
Correlations between the thickness of the neutron skin in finite nuclei and the nuclear matter symmetry energy are studied in the Skyrme Hartree-Fock model. From the most recent analysis of the isospin diffusion data in heavy-ion collisions based on an isospin- and momentum-dependent transport model with in-medium nucleonnucleon cross sections, a value of $L=88 \pm 25 \mathrm{MeV}$ for the slope of the nuclear symmetry energy at saturation density is extracted, and this imposes stringent constraints on both the parameters in the Skyrme effective interactions and the neutron skin thickness of heavy nuclei. Predicted thickness of the neutron skin is $0.22 \pm$ $0.04 \mathrm{fm}$ for ${ }^{208} \mathrm{~Pb}, 0.29 \pm 0.04 \mathrm{fm}$ for ${ }^{132} \mathrm{Sn}$, and $0.22 \pm 0.04 \mathrm{fm}$ for ${ }^{124} \mathrm{Sn}$.
\end{abstract}

DOI: 10.1103/PhysRevC.72.064309

PACS number(s): 25.70.-z, 21.30.Fe, 21.10.Gv

\section{INTRODUCTION}

The study of the equation of state (EOS) of isospin asymmetric nuclear matter, especially the nuclear symmetry energy, is currently an active field of research in nuclear physics [1-8]. Although the nuclear symmetry energy at normal nuclear matter density is known to be around $30 \mathrm{MeV}$ from the empirical liquid-drop mass formula [9,10], its values at other densities, especially at supranormal densities, are poorly known $[1,2]$. Advances in radioactive nuclear beam facilities provide, however, the possibility to pin down the density dependence of the nuclear symmetry energy in heavy ion collisions induced by these nuclei $[1,2,7,11-34]$. Indeed, significant progress has recently been made in extracting the information on the density dependence of nuclear symmetry energy from the isospin diffusion data in heavy-ion collisions from the NSCL/MSU [35-37]. Using an isospinand momentum-dependent IBUU04 transport model with in-medium nucleon-nucleon $(N N)$ cross sections, the isospin diffusion data were found to be consistent with a relatively soft nuclear symmetry energy at subnormal density [37].

Information on the density dependence of the nuclear symmetry energy can in principle also be obtained from the thickness of the neutron skin in heavy nuclei as the latter is strongly correlated with the slope $L$ of the nuclear matter symmetry energy at saturation density [4,38-42]. Because of the large uncertainties in measured neutron skin thickness of heavy nuclei, this has not been possible. Instead, studies have been carried out to use the extracted nuclear symmetry energy from the isospin diffusion data to constrain the neutron skin thickness of heavy nuclei $[37,43]$. Using the Hartree-Fock approximation with parameters fitted to the phenomenological EOS that was used in the IBUU04 transport model to describe the isospin diffusion data from the NSCL/MSU, it was found that a neutron skin thickness of less than $0.15 \mathrm{fm}[37,43]$ for ${ }^{208} \mathrm{~Pb}$ was incompatible with the isospin diffusion data.

In the present work, we study more systematically the correlation between the density dependence of the nuclear symmetry energy and the thickness of the neutron skin in a number of nuclei within the framework of the Skyrme Hartree-Fock model. Extracting the values of $L$ from the most recently determined density dependence of the nuclear symmetry energy from the isospin diffusion data in heavy-ion collisions, we obtain stringent constraints on the neutron skin thickness of the nuclei ${ }^{208} \mathrm{~Pb},{ }^{132} \mathrm{Sn}$, and ${ }^{124} \mathrm{Sn}$. The extracted value of $L$ also limits the allowed parameter sets for the Skyrme interaction.

\section{NUCLEAR SYMMETRY ENERGY AND THE SKYRME INTERACTIONS}

The nuclear symmetry energy $E_{\text {sym }}(\rho)$ at nuclear density $\rho$ can be expanded around the nuclear matter saturation density $\rho_{0}$ as

$$
E_{\text {sym }}(\rho)=E_{\text {sym }}\left(\rho_{0}\right)+\frac{L}{3}\left(\frac{\rho-\rho_{0}}{\rho_{0}}\right)+\frac{K_{\text {sym }}}{18}\left(\frac{\rho-\rho_{0}}{\rho_{0}}\right)^{2},
$$

where $L$ and $K_{\text {sym }}$ are the slope and curvature of the nuclear symmetry energy at $\rho_{0}$, i.e.,

$$
\begin{aligned}
L & =\left.3 \rho_{0} \frac{\partial E_{\mathrm{sym}}(\rho)}{\partial \rho}\right|_{\rho=\rho_{0}}, \\
K_{\mathrm{sym}} & =\left.9 \rho_{0}^{2} \frac{\partial^{2} E_{\mathrm{sym}}(\rho)}{\partial^{2} \rho}\right|_{\rho=\rho_{0}} .
\end{aligned}
$$

The $L$ and $K_{\text {sym }}$ characterize the density dependence of the nuclear symmetry energy around normal nuclear matter density and thus provide important information on the properties of nuclear symmetry energy at both high and low densities.

In the standard Skyrme Hartree-Fock model [38,44-48], the interaction is taken to have a zero-range, density- and momentum-dependent form and the Skyrme interaction parameters are chosen to fit the binding energies and charge radii of a large number of nuclei in the periodic table. For infinite nuclear matter, the nuclear symmetry energy from the Skyrme 


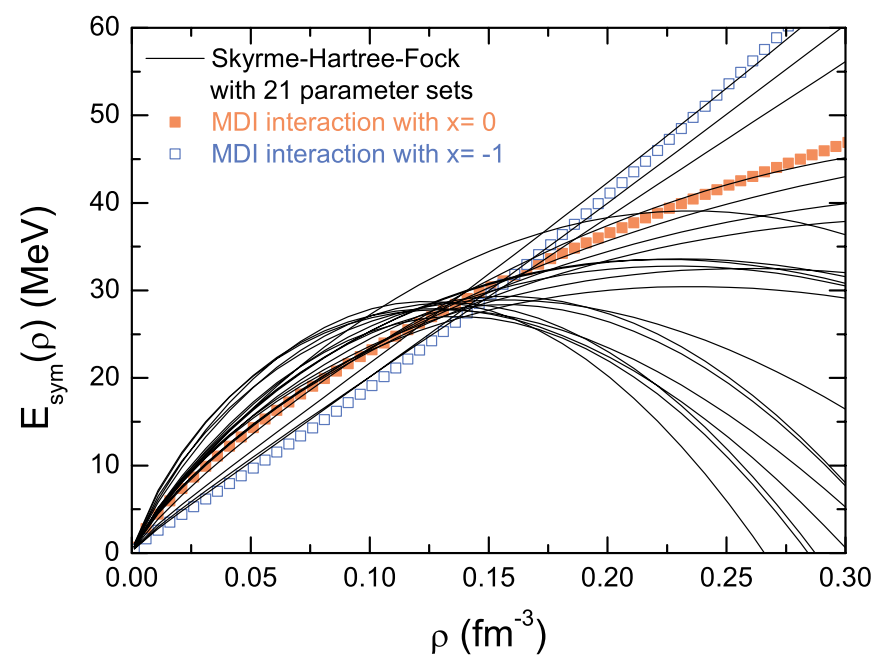

FIG. 1. (Color online) Density dependence of the nuclear symmetry energy $E_{\text {sym }}(\rho)$ for 21 sets of Skyrme interaction parameters. The results from the MDI interaction with $x=-1$ (open squares) and 0 (solid squares) are also shown.

interaction can be expressed as follows $[47,48]$ :

$$
\begin{aligned}
E_{\text {sym }}(\rho)= & \frac{1}{3} \frac{\hbar^{2}}{2 m}\left(\frac{3 \pi^{2}}{2}\right)^{2 / 3} \rho^{2 / 3}-\frac{1}{8} t_{0}\left(2 x_{0}+1\right) \rho \\
& -\frac{1}{48} t_{3}\left(2 x_{3}+1\right) \rho^{\sigma+1}+\frac{1}{24}\left(\frac{3 \pi^{2}}{2}\right)^{2 / 3} \\
& \times\left[-3 t_{1} x_{1}+\left(4+5 x_{2}\right) t_{2}\right] \rho^{5 / 3},
\end{aligned}
$$

where the $\sigma, t_{0}-t_{3}$, and $x_{0}-x_{3}$ are Skyrme interaction parameters.

Figure 1 displays the density dependence of $E_{\text {sym }}(\rho)$ for 21 sets of Skyrme interaction parameters, i.e., SKM, SKM ${ }^{*}$ RATP, SI, SII, SIII, SIV, SV, SVI, $E, E_{\sigma}$, $G_{\sigma}, R_{\sigma}, Z, Z_{\sigma}, Z_{\sigma}^{*}, T, \mathrm{~T} 3, \mathrm{SkX}$, SkXce, and SkXm. Values of the parameters in these Skyrme interactions can be found in Refs. [44-46]. For comparison, we also show in Fig. 1 results from the phenomenological parametrization of the momentum-dependent nuclear mean-field potential based on the Gogny effective interaction [49], i.e., the MDI interactions with $x=-1$ (open squares) and 0 (solid squares), where different $x$ values correspond to different density dependence of the nuclear symmetry energy but keep other properties of the nuclear EOS the same [36]. From comparing the isospin diffusion data from NSCL/MSU using the IBUU04 transport model with in-medium $N N$ cross sections, these interactions are recently shown to give, respectively, the upper and lower bounds for the stiffness of the nuclear symmetry energy [37]. It is seen from Fig. 1 that the density dependence of the symmetry energy varies drastically among different interactions. Although the values of $E_{\text {sym }}\left(\rho_{0}\right)$ are all in the range of $26-35 \mathrm{MeV}$, the values of $L$ and $K_{\text {sym }}$ are in the range of $-50-100 \mathrm{MeV}$ and $-700-50 \mathrm{MeV}$, respectively.

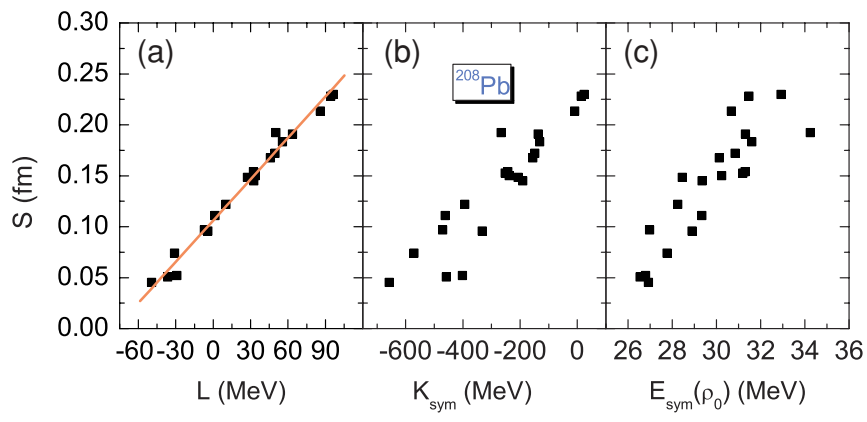

FIG. 2. (Color online) Neutron skin thickness $S$ of ${ }^{208} \mathrm{~Pb}$ as a function of (a) $L$, (b) $K_{\text {sym }}$, and (c) $E_{\text {sym }}\left(\rho_{0}\right)$ for 21 sets of Skyrme interaction parameters. The line in panel (a) represents a linear fit.

\section{NEUTRON SKIN THICKNESS OF FINITE NUCLEI AND THE SLOPE OF NUCLEAR SYMMETRY ENERGY AT SATURATION DENSITY}

The neutron skin thickness $S$ of a nucleus is defined as the difference between the root-mean-square radii $\sqrt{\left\langle r_{n}\right\rangle}$ of neutrons and $\sqrt{\left\langle r_{p}\right\rangle}$ of protons, i.e.,

$$
S=\sqrt{\left\langle r_{n}^{2}\right\rangle}-\sqrt{\left\langle r_{p}^{2}\right\rangle}
$$

It has been known that $S$ is sensitive to the density dependence of the nuclear symmetry energy, particularly the slope parameter $L$ at the normal nuclear matter density [4,38-42]. Using above 21 sets of Skyrme interaction parameters, we have evaluated the neutron skin thickness of several nuclei. In Figs. 2(a), 2(b), and 2(c), we show, respectively, the correlations between the neutron skin thickness of ${ }^{208} \mathrm{~Pb}$ with $L, K_{\text {sym }}$, and $E_{\text {sym }}\left(\rho_{0}\right)$. It is seen from Fig. 2(a) that there exists an approximate linear correlation between $S$ and $L$. The correlations of $S$ with $K_{\text {sym }}$ and $E_{\text {sym }}\left(\rho_{0}\right)$ are less strong and even exhibit some irregular behavior. The solid line in Fig. 2(a) is a linear fit to the correlation between $S$ and $L$ and is given by the following expression:

$$
\begin{aligned}
S\left({ }^{208} \mathrm{~Pb}\right)= & (0.1066 \pm 0.0019) \\
& +\left(0.00133 \pm 3.76 \times 10^{-5}\right) \times L
\end{aligned}
$$

or

$$
L=(-78.5 \pm 3.2)+(740.4 \pm 20.9) \times S\left({ }^{208} \mathrm{~Pb}\right),
$$

where the units of $L$ and $S$ are $\mathrm{MeV}$ and fm, respectively. Therefore, if the value for either $S\left({ }^{208} \mathrm{~Pb}\right)$ or $L$ is known, the value for the other can be determined.

It is of interest to see if there are also correlations between the neutron skin thickness of other neutron-rich nuclei and the nuclear symmetry energy. Figure 3 shows the same correlations as in Fig. 2 but for the neutron-rich nuclei ${ }^{132} \mathrm{Sn},{ }^{124} \mathrm{Sn}$, and ${ }^{48} \mathrm{Ca}$. For the heavy ${ }^{132} \mathrm{Sn}$ and ${ }^{124} \mathrm{Sn}$, we obtain a similar conclusion as for ${ }^{208} \mathrm{~Pb}$, namely $S$ exhibits an approximate linear correlation with $L$ but weaker correlations with $K_{\text {sym }}$ and $E_{\text {sym }}\left(\rho_{0}\right)$. For the lighter ${ }^{48} \mathrm{Ca}$, however, all the correlations become weaker than those of heavier nuclei. Therefore, the neutron skin thickness of heavy nuclei is better correlated with the density dependence of the nuclear 


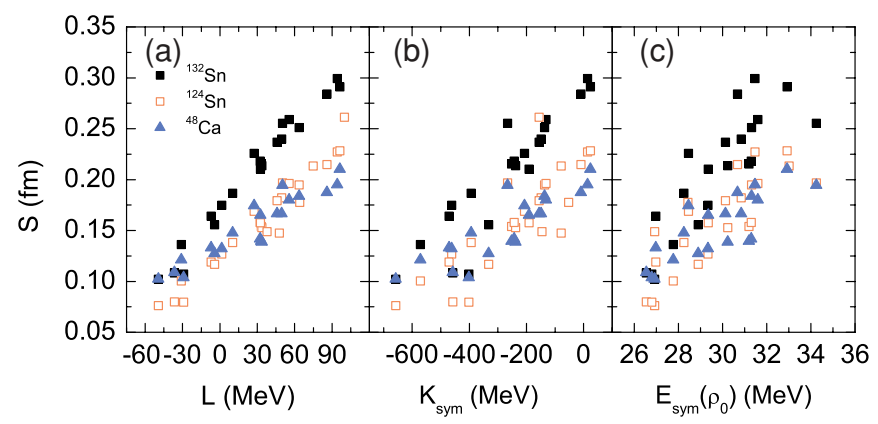

FIG. 3. (Color online) Same as Fig. 2 but for nuclei ${ }^{132} \mathrm{Sn}$ (solid squares), ${ }^{124} \mathrm{Sn}$ (open squares), and ${ }^{48} \mathrm{Ca}$ (triangles).

symmetry energy. As in Eq. (6) and (7), a linear fit to the correlation between $S$ and $L$ can also be obtained for ${ }^{132} \mathrm{Sn}$ and ${ }^{124} \mathrm{Sn}$, and the corresponding expressions are as follows:

$$
\begin{aligned}
S\left({ }^{132} \mathrm{Sn}\right)= & (0.1694 \pm 0.0025) \\
& +\left(0.0014 \pm 5.12 \times 10^{-5}\right) \times L, \\
L= & (-117.1 \pm 5.4) \\
& +(695.1 \pm 25.3) \times S\left({ }^{132} \mathrm{Sn}\right),
\end{aligned}
$$

and

$$
\begin{aligned}
S\left({ }^{124} \mathrm{Sn}\right)= & (0.1255 \pm 0.0020) \\
& +\left(0.0011 \pm 4.05 \times 10^{-5}\right) \times L, \\
L= & (-110.1 \pm 5.2) \\
& +(882.6 \pm 32.3) \times S\left({ }^{124} \mathrm{Sn}\right) .
\end{aligned}
$$

Similar linear relations between $S$ and $L$ are also expected for other heavy nuclei. This is not surprising as detailed discussions in Refs. [4,38-42] have shown that the thickness of the neutron skin in heavy nuclei is determined by the pressure difference between neutrons and protons, which is proportional to the parameter $L$.

To give a quantitative estimate of above discussed correlations, we define the following linear correlation coefficient $C_{l}$ :

$$
C_{l}=\sqrt{1-q / t}
$$

where

$$
\begin{aligned}
q & =\sum_{i=1}^{n}\left[y_{i}-\left(A+B x_{i}\right)\right]^{2}, \\
t & =\sum_{i=1}^{n}\left(y_{i}-\bar{y}\right), \quad \bar{y}=\sum_{i=1}^{n} y_{i} / n .
\end{aligned}
$$

In the above, $A$ and $B$ are the linear regression coefficients, $\left(x_{i}, y_{i}\right)$ are the sample points and $n$ is the number of sample points. The linear correlation coefficient $C_{l}$ measures the degree of linear correlation, and $C_{l}=1$ corresponds to an ideal linear correlation. Table I gives the linear correlation coefficient $C_{l}$ for the correlation of $S$ with $L, K_{\text {sym }}$, and $E_{\text {sym }}\left(\rho_{0}\right)$ for ${ }^{208} \mathrm{~Pb},{ }^{132} \mathrm{Sn},{ }^{124} \mathrm{Sn}$, and ${ }^{48} \mathrm{Ca}$ shown in Figs. 2 and 3 for different Skyrme interactions. It is seen that these
TABLE I. Linear correlation coefficients $C_{l}$ of $S$ with $L, K_{\mathrm{sym}}$, and $E_{\text {sym }}\left(\rho_{0}\right)$ for ${ }^{208} \mathrm{~Pb},{ }^{132} \mathrm{Sn},{ }^{124} \mathrm{Sn}$, and ${ }^{48} \mathrm{Ca}$ from 21 sets of Skyrme interaction parameters.

\begin{tabular}{lcccc}
\hline \hline$C_{l}(\%)$ & ${ }^{208} \mathrm{~Pb}$ & ${ }^{132} \mathrm{Sn}$ & ${ }^{124} \mathrm{Sn}$ & ${ }^{48} \mathrm{Ca}$ \\
\hline$S-L$ & 99.25 & 98.76 & 98.75 & 93.66 \\
$S-K_{\text {sym }}$ & 92.26 & 92.06 & 92.22 & 86.99 \\
$S-E_{\text {sym }}$ & 87.89 & 85.74 & 85.77 & 81.01 \\
\hline \hline
\end{tabular}

correlations become weaker with decreasing nucleus mass, and a strong linear correlation only exists between the $S$ and $L$ for the heavier nuclei ${ }^{208} \mathrm{~Pb},{ }^{132} \mathrm{Sn}$, and ${ }^{124} \mathrm{Sn}$. Therefore, the neutron skin thickness of these nuclei can be extracted once the slope parameter $L$ of the nuclear symmetry energy at saturation density is known.

\section{CONSTRAINTS FROM ISOSPIN DIFFUSION DATA IN HEAVY-ION COLLISIONS}

Experimentally, the degree of isospin diffusion between the projectile nucleus $A$ and the target nucleus $B$ can be studied via the quantity $R_{i}[35,50]$,

$$
R_{i}=\frac{2 X^{A+B}-X^{A+A}-X^{B+B}}{X^{A+A}-X^{B+B}},
$$

where $X$ is any isospin-sensitive observable. By construction, the value of $R_{i}$ is $1(-1)$ for symmetric $A+A(B+B)$ reaction. If isospin equilibrium is reached during the collision as a result of isospin diffusion, the value of $R_{i}$ is about zero. In the NSCL/MSU experiments with $A={ }^{124} \mathrm{Sn}$ and $B={ }^{112} \mathrm{Sn}$ at a beam energy of $50 \mathrm{MeV} /$ nucleon and an impact parameter about $6 \mathrm{fm}$, the isospin asymmetry of the projectile-like residue was used as the isospin tracer $X$ [35]. Using an isospinand momentum-dependent IBUU04 transport model with free-space experimental $N N$ cross sections, the dependence of $R_{i}$ on the nuclear symmetry energy was studied from the average isospin asymmetry of the projectile-like residue that was calculated from nucleons with local densities higher than $\rho_{0} / 20$ and velocities larger than $1 / 2$ the beam velocity in the center-of-mass frame [36]. Comparing the theoretical results with the experimental data has allowed us to extract a nuclear symmetry energy of $E_{\text {sym }}(\rho) \approx 31.6\left(\rho / \rho_{0}\right)^{1.05}$. Including also medium-dependent $N N$ cross sections, which are important for isospin-dependent observables [37,51], the isospin diffusion data leads to an even softer nuclear symmetry energy of $E_{\text {sym }}(\rho) \approx 31.6\left(\rho / \rho_{0}\right)^{0.7}[37]$.

In Fig. 4, we show the results from the IBUU04 transport model with in-medium $N N$ cross sections, that are consistent with the mean-field potential obtained with the MDI interactions used in the model, for the degree of the isospin diffusion $1-R_{i}$ as a function of $L$. The shaded band in Fig. 4 indicates the data from NSCL/MSU [35]. It is seen that the strength of isospin diffusion $1-R_{i}$ decreases monotonically with decreasing value of $x$ or increasing value of $L$. This is expected as the parameter $L$ reflects the difference in the pressures on neutrons and protons. From comparison of 


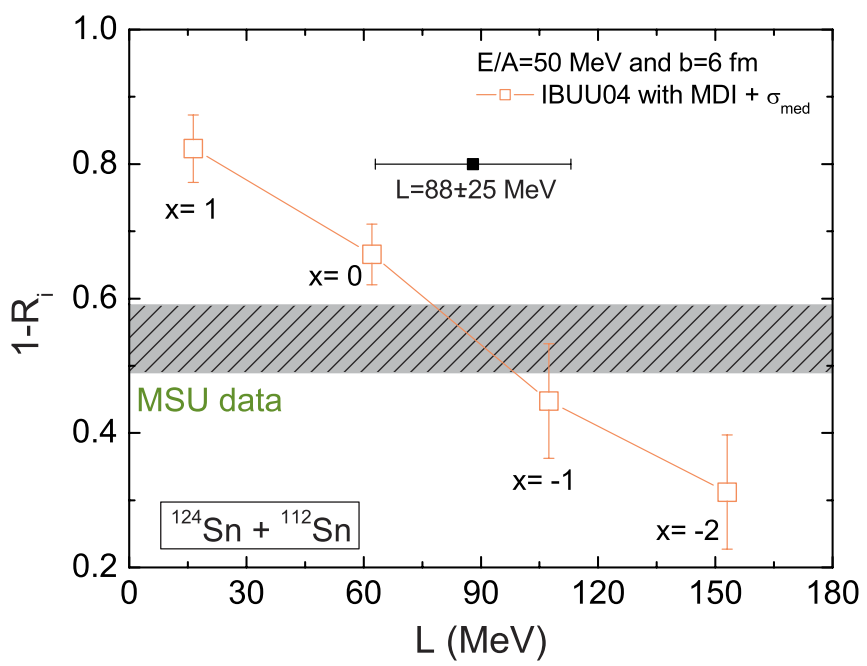

FIG. 4. (Color online) Degree of the isospin diffusion $1-R_{i}$ as a function of $L$ using the MDI interaction with $x=-2,-1,0$, and 1 . The shaded band indicates the data from NSCL/MSU [35]. The solid square with error bar represents $L=88 \pm 25 \mathrm{MeV}$.

the theoretical results with the data, we can clearly exclude the MDI interaction with $x=1$ and $x=-2$ as they give either too large or too small a value for $1-R_{i}$ compared to that of data. The range of $x$ or $L$ values that give values of $1-R_{i}$ falling within the band of experimental values could in principle be determined in our model by detailed calculations. Instead, we determine this schematically by using the results from the four $x$ values. For the centroid value of $L$, it is obtained from the interception of the line connecting the theoretical results at $x=-1$ and 0 with the central value of $1-R_{i}$ data in Fig. 4, i.e., $L=88 \mathrm{MeV}$. The upper limit of $L=113 \mathrm{MeV}$ is estimated from the interception of the line connecting the upper error bars of the theoretical results at $x=-1$ and -2 with the lower limit of the data band of $1-R_{i}$. Similarly, the lower limit of $L=65 \mathrm{MeV}$ is estimated from the interception of the line connecting the lower error bars of the theoretical results at $x=0$ and -1 with the upper limit of the data band of $1-R_{i}$. This leads to an extracted value of $L=$ $88 \pm 25 \mathrm{MeV}$ as shown by the solid square with error bar in Fig. 4.

The extracted value of $L=88 \pm 25 \mathrm{MeV}$ gives a rather stringent constraint on the density dependence of the nuclear symmetry energy and thus puts strong constraints on the nuclear effective interactions as well. For the Skyrme effective interactions shown in Fig. 1, for instance, all of those lie beyond $x=0$ and $x=-1$ in the subsaturation region are not consistent with the extracted value of $L$. Actually, we note that only four sets of Skyrme interactions, i.e., SIV, SV, $G_{\sigma}$, and $R_{\sigma}$, in the 21 sets of Skyrme interactions considered here have nuclear symmetry energies that are consistent with the extracted $L$ value.

The extracted $L$ value also allows us to determine from Eqs. (6), (8), and (10), a neutron skin thickness of $0.22 \pm$ $0.04 \mathrm{fm}$ for ${ }^{208} \mathrm{~Pb}, 0.29 \pm 0.04 \mathrm{fm}$ for ${ }^{132} \mathrm{Sn}$, and $0.22 \pm 0.04$ $\mathrm{fm}$ for ${ }^{124} \mathrm{Sn}$. Experimentally, great efforts were devoted to measure the thickness of the neutron skin in heavy nuclei [52,53], and a recent review can be found in Ref. [54]. The data for the neutron skin thickness of ${ }^{208} \mathrm{~Pb}$ indicate a large uncertainty, i.e., $0.1-0.28 \mathrm{fm}$. Our results for the neutron skin thickness of ${ }^{208} \mathrm{~Pb}$ are thus consistent with present data but give a much stronger constraint. A large uncertainty is also found experimentally in the neutron skin thickness of ${ }^{124} \mathrm{Sn}$, i.e., its value varies from 0.1 to $0.3 \mathrm{fm}$ depending on the experimental method. The proposed experiment of parity-violating electron scattering from ${ }^{208} \mathrm{~Pb}$ at the Jefferson Laboratory is expected to give another independent and more accurate measurement of its neutron skin thickness (within $0.05 \mathrm{fm}$ ), thus providing improved constraints on the density dependence of the nuclear symmetry energy $[55,56]$.

Most recently, an accurately calibrated relativistic parametrization based on the relativistic mean-field theory has been introduced to study the neutron skin thickness of finite nuclei [57]. This parametrization can describe simultaneously the ground-state properties of finite nuclei and their monopole and dipole resonances. Using this parametrization, the authors predicted a neutron skin thickness of $0.21 \mathrm{fm}$ in ${ }^{208} \mathrm{~Pb}, 0.27 \mathrm{fm}$ in ${ }^{132} \mathrm{Sn}$, and $0.19 \mathrm{fm}$ in ${ }^{124} \mathrm{Sn}[57,58]$. These predictions are in surprisingly good agreement with our results constrained by the isospin diffusion data in heavy-ion collisions.

\section{SUMMARY}

In summary, we have studied the correlation between the neutron skin thickness of finite nuclei and the nuclear symmetry energy within the framework of the Skyrme HartreeFock model. As in previous studies, we have found a strong linear correlation between the neutron skin thickness of heavy nuclei and the slope $L$ of the nuclear matter symmetry energy at saturation density. This correlation provides stringent constraints on both the density dependence of the nuclear symmetry energy and the thickness of the neutron skin in heavy nuclei. From the most recent analysis of the isospin diffusion data in heavy-ion collisions using an isospinand momentum-dependent transport model with in-medium $N N$ cross sections, the value $L=88 \pm 25 \mathrm{MeV}$ has been extracted. The relatively constrained value for the slope of the nuclear matter symmetry energy imposes strong constraints on the parameters in the Skyrme effective interactions and leads to predicted neutron skin thickness of $0.22 \pm 0.04 \mathrm{fm}$ for ${ }^{208} \mathrm{~Pb}, 0.29 \pm 0.04 \mathrm{fm}$ for ${ }^{132} \mathrm{Sn}$, and $0.22 \pm 0.04 \mathrm{fm}$ for ${ }^{124} \mathrm{Sn}$.

\section{ACKNOWLEDGMENTS}

The work was supported in part by the National Natural Science Foundation of China under grant 10105008 and 10575071 (LWC), the U.S. National Science Foundation under grant PHY-0457265 and the Welch Foundation under grant A-1358 (CMK), as well as the U.S. National Science Foundation under grant PHY-0354572 and PHY-0456890 and the NASA-Arkansas Space Grants Consortium Award ASU15154 (BAL). 
[1] B. A. Li, C. M. Ko, and W. Bauer, topical review, Int. J. Mod. Phys. E 7, 147 (1998).

[2] Isospin Physics in Heavy-Ion Collisions at Intermediate Energies, edited by Bao-An Li and W. Udo Schröder (Nova Science Publishers, New York, 2001).

[3] I. Bombbaci, Isospin Physics in Heavy-Ion Collisions at Intermediate Energies, edited by Bao-An Li and W. Udo Schröder (Nova Science, New York, 2001), p. 35.

[4] A. E. L. Dieperink, Y. Dewulf, D. Van Neck, M. Waroquier, and V. Rodin, Phys. Rev. C 68, 064307 (2003).

[5] P. Danielewicz, R. Lacey, and W. G. Lynch, Science 298, 1592 (2002).

[6] J. M. Lattimer and M. Prakash, Science 304, 536 (2004).

[7] V. Baran, M. Colonna, V. Greco, and M. Di Toro, Phys. Rep. 410, 335 (2005).

[8] A. W. Steiner, M. Prakash, J. M. Lattimer, and P. J. Ellis, Phys. Rep. 411, 325 (2005).

[9] W. D. Myers and W. J. Swiatecki, Nucl. Phys. A81, 1 (1966).

[10] K. Pomorski and J. Dudek, Phys. Rev. C 67, 044316 (2003).

[11] B. A. Li, C. M. Ko, and Z. Z. Ren, Phys. Rev. Lett. 78, 1644 (1997).

[12] H. Muller and B. D. Serot, Phys. Rev. C 52, 2072 (1995).

[13] B. A. Li and C. M. Ko, Nucl. Phys. A618, 498 (1997).

[14] V. Baran, M. Colonna, M. Di Toro, and A. B. Larionov, Nucl. Phys. A632, 287 (1998).

[15] H. S. Xu et al., Phys. Rev. Lett. 85, 716 (2000).

[16] W. P. Tan et al., Phys. Rev. C 64, 051901(R) (2001).

[17] V. Baran, M. Colonna, M. Di Toro, V. Greco, M.Zielinska-Pfabé, and H. H. Wolter, Nucl. Phys. A703, 603 (2002).

[18] M. B. Tsang, W. A. Friedman, C. K. Gelbke, W. G. Lynch, G. Verde, and H. Xu, Phys. Rev. Lett. 86, 5023 (2001).

[19] B. A. Li, A. T. Sustich, and B. Zhang, Phys. Rev. C 64, 054604 (2001).

[20] B. A. Li, Phys. Rev. Lett. 85, 4221 (2000).

[21] Special issue on Radioactive Nuclear Beams, edited by I. Tanihata [Nucl. Phys. A693 (2001)].

[22] B. A. Li, Phys. Rev. Lett. 88, 192701 (2002); Nucl. Phys. A708, 365 (2002).

[23] L. W. Chen, V. Greco, C. M. Ko, and B. A. Li, Phys. Rev. Lett. 90, 162701 (2003); Phys. Rev. C 68, 014605 (2003); L. W. Chen, C. M. Ko, and B. A. Li, ibid. 68, 017601 (2003); Nucl. Phys. A729, 809 (2003).

[24] A. Ono, P. Danielewicz, W. A. Friedman, W. G. Lynch, and M. B. Tsang, Phys. Rev. C 68, 051601(R) (2003).

[25] J. Y. Liu, W. J. Guo, Y. Z. Xing, and H. Liu, Nucl. Phys. A726, $123(2003)$.

[26] L. W. Chen, C. M. Ko, and B. A. Li, Phys. Rev. C 69, 054606 (2004).

[27] B. A. Li, C. B. Das, S. Das Gupta, and C. Gale, Phys. Rev. C 69, 011603(R) (2004); Nucl. Phys. A735, 563 (2004).
[28] L. Shi and P. Danielewicz, Phys. Rev. C 68, 064604 (2003).

[29] B. A. Li, Phys. Rev. C 69, 034614 (2004).

[30] J. Rizzo, M. Colonna, M. Di Toro, and V. Greco, Nucl. Phys. A732, 202 (2004)

[31] B. A. Li, G. C. Yong, and W. Zuo, Phys. Rev. C 71, 014608 (2005).

[32] Y. Zhang and Z. Li, Phys. Rev. C 71, 024604 (2005).

[33] Q. Li, Z. Li, E. Zhao, and R. K. Gupta, Phys. Rev. C 71, 054907 (2005).

[34] W. D. Tian et al., Chin. Phys. Lett. 22, 306 (2005).

[35] M. B. Tsang et al., Phys. Rev. Lett. 92, 062701 (2004).

[36] L. W. Chen, C. M. Ko, and B. A. Li, Phys. Rev. Lett. 94, 032701 (2005).

[37] B. A. Li and L. W. Chen, Phys. Rev. C (2005), in press; [nuclth/0508024].

[38] B. A. Brown, Phys. Rev. Lett. 85, 5296 (2000).

[39] C. J. Horowitz and J. Piekarewicz, Phys. Rev. Lett. 86, 5647 (2001); Phys. Rev. C 66, 055803 (2002).

[40] S. Typel and B. A. Brown, Phys. Rev. C 64, 027302 (2001).

[41] R. J. Furnstahl, Nucl. Phys. A706, 85 (2002).

[42] S. Karataglidis, K. Amos, B. A. Brown, and P. K. Deb, Phys. Rev. C 65, 044306 (2002).

[43] A. W. Steiner and B. A. Li, Phys. Rev. C 72, 041601(R) (2005).

[44] M. Brack, C. Guet, and H.-B. Hakansson, Phys. Rep. 123, 275 (1985).

[45] J. Friedrich and P.-G. Reinhard, Phys. Rev. C 33, 335 (1986).

[46] B. A. Brown, Phys. Rev. C 58, 220 (1998).

[47] L. W. Chen and F. S. Zhang, High Energy Phys. Nucl. Phys. 23, 1197 (1999) (in Chinese).

[48] J. R. Stone, J. C. Miller, R. Koncewicz, P. D. Stevenson, and M. R. Strayer, Phys. Rev. C 68, 034324 (2003).

[49] C. B. Das, S. Das Gupta, C. Gale, and B. A. Li, Phys. Rev. C 67, 034611 (2003).

[50] F. Rami et al., Phys. Rev. Lett. 84, 1120 (2000).

[51] B. A. Li, G. C. Yong, and W. Zuo, Phys. Rev. C 71, 044604 (2005).

[52] V. E. Starodubsky and N. M. Hintz, Phys. Rev. C 49, 2118 (1994).

[53] B. C. Clark, L. J. Kerr, and S. Hama, Phys. Rev. C 67, 054605 (2003).

[54] A. Krasznahorkay et al., Nucl. Phys. A731, 224 (2004).

[55] C. J. Horowitz, S. J. Pollock, P. A. Souder, and R. Michaels, Phys. Rev. C 63, 025501 (2001).

[56] R. Michaels, P. A. Souder, and G. M. Urciuoli, Thomas Jefferson National Accelerator Facility Proposal E-00-003, 2002, http:// www.jlab.org/exp_prog/generated/halla.html.

[57] B. G. Todd-Rutel and J. Piekarewicz, Phys. Rev. Lett. 95, 122501 (2005).

[58] J. Piekarewicz (Private communications). 